\section{ISOTERMAS DE EQUILÍBRIO E TERMODINÂMICA DA ADSORÇÃO DO CORANTE TARTRAZINA UTILIZANDO ESFERAS RECOBERTAS COM QUITOSANA}

\author{
W. S. MARTINS ${ }^{1}$, V. A. VIEIRA ${ }^{1}$, J. F. LOPES $^{1}$, T. PIVA ${ }^{1}$, M. L. G. VIEIRA ${ }^{1}$, L. A. (A) \\ PINTO $^{1}$
}

${ }^{1}$ Universidade Federal do Rio Grande, Escola de Química e Alimentos

E-mail para contato: winnetousoaresmartins@gmail.com

\begin{abstract}
RESUMO - Um adsorvente promissor é a quitosana, esta tem mostrado resultados satisfatórios para a adsorção de corantes. A quitosana pode ser modificada para melhorar as suas propriedades. Para adsorção em leito fixo se mostra interessante a imobilização da quitosana em material inerte com a finalidade de aumentar a resistência mecânica. Este trabalho teve como objetivo o estudo das isotermas de equilíbrio para a adsorção do corante tartrazina utilizando esferas recobertas por quitosana. Verificou-se o efeito da temperatura ( $293 \mathrm{~K}$ à $333 \mathrm{~K})$, os dados experimentais de equilíbrio foram correlacionados com o modelo de BET e estimaram-se as variações da energia de Gibbs $(\Delta \mathrm{G})$, entalpia $(\Delta \mathrm{H})$ e entropia $(\Delta \mathrm{S})$ para o processo. A adsorção do corante tartrazina foi favorecida pelo aumento da temperatura. O modelo de BET foi adequado para representar os dados experimentais de equilíbrio $\left(\mathrm{R}^{2}>0,95\right.$ e $\left.\mathrm{EMR}<5 \%\right)$, sendo que a máxima capacidade de adsorção na monocamada foi de $153,3 \mathrm{mg} \mathrm{g}^{-1}$ obtida a $313 \mathrm{~K}$. Os valores de $\Delta \mathrm{G}, \Delta \mathrm{H}$ e $\Delta \mathrm{S}$ indicaram que a adsorção do corante tartrazina por esferas recobertas com quitosana foi espontânea, favorável, endotérmica.
\end{abstract}

\section{INTRODUÇÃO}

Os corantes são utilizados em diversos setores industriais, como na indústria têxtil, de cosméticos, de papel, de processamento de alimentícios e de fármacos (Crini e Badot, 2008). De acordo com a classificação no Color Index existem mais de 10.000 variedades de corantes sintetizados e disponíveis a estas indústrias (Moussavi et al., 2009). Devido à baixa taxa de fixação destes corantes é gerada elevada quantidade de efluentes líquidos (Dotto et al., 2011). Algumas tecnologias utilizadas para tratamento de efluentes como, por exemplo, floculação combinada com flotação, filtração por membranas, destruição eletroquímica e irradiação, apresentam baixas taxas de remoção, além do custo elevado (Srinivasan e Viraraghavan, 2010). Entretanto, a adsorção vem se mostrando bastante eficiente para o tratamento destes efluentes (Crini e Badot, 2008; Dotto et al., 2011; Vieira et al., 2014). O carvão ativado é o adsorvente mais utilizado, mas este apresenta um alto custo em aplicações de larga escala (Shirzad-Siboni et al.,2014). Desta forma, surge a necessidade de aplicação de outros tipos de adsorventes, por exemplo, a utilização de quitosana, que é derivada de recursos naturais e possui um alto custo-benefício (Srinivasan e Viraraghavan, 2010). Uma das principais características da adsorção pela quitosana é o grupo amina primária, o qual é protonado para
Comentar [U]: Os quatro primeiros autores são iniciantes científicos e participaram tanto da parte experimental quanto do tratamento de dados, discussão dos resultados e elaboração do trabalho. Os mesmos realizaram estas atividades supervisionados por aluna de doutorado e orientador. 


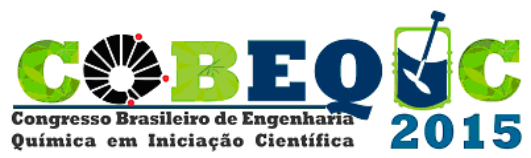

XI Congresso Brasileiro de Engenharia Química em Iniciação Científica Unicamp - Campinas - SP 19 a 22 de julho de 2015

formar $-\mathrm{NH}_{3}^{+}$em soluções ácidas, que irá interagir com corantes aniônicos (Chiou et al, 2004).

$\mathrm{Na}$ adsorção é imprescindível o conhecimento do comportamento do adsorvente frente ao adsorbato. Estas informações podem ser fornecidas através das isotermas de equilíbrio, as quais estabelecem a relação entre a quantidade de corante adsorvida pelo adsorvente e a quantidade na fase líquida. Além disso, os mecanismos de adsorção podem ser estudados e interpretados por parâmetros termodinâmicos (Blázquez et al., 2010). Este trabalho teve como objetivo o estudo da adsorção do corante tartrazina utilizando esferas recobertas por quitosana através das isotermas de equilíbrio. Foi avaliado o efeito da temperatura (293 K à 313 [ K $)_{2}$ a correlação dos dados experimentais de equilíbrio com o modelo de BET e calculados os parâmetros termodinâmicos de energia de Gibbs $(\Delta \mathrm{G})$, entalpia $(\Delta \mathrm{H})$ e entropia $(\Delta \mathrm{S})$.

\section{MATERIAL E MÉTODOS}

\subsection{Adsorbato}

Foi utilizado o corante tartrazina, massa molar 534,4 g.mol ${ }^{-1}$, pureza $60 \% \%$ e comprimento de onda de máxima absorção de $425 \mathrm{~nm}$. O corante foi fornecido pela Indústria Duas Rodas, Brasil. A estrutura química do corante está apresentada na Figura 1.

Figura 1 - Estrutura química do corante tartrazina (Dotto et al., 2011).

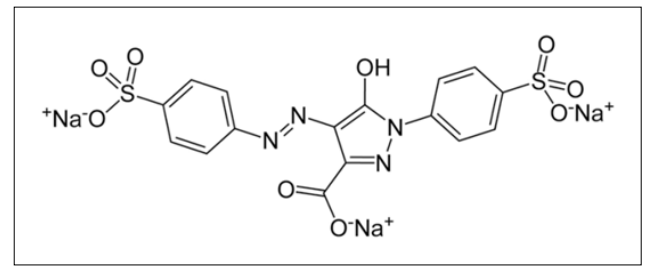

\subsection{Recobrimento das esferas}

As esferas de vidro ( $1 \mathrm{~mm}$ de diâmetro, densidade $2300 \mathrm{~kg} . \mathrm{m}^{-3}$ e esfericidade de 0,99 ) foram adquiridas da Nacional Esferas Ltda, Brasil. O recobrimento das esferas por quitosana foi realizado através da técnica de dip coating. As esferas foram imersas na solução de quitosana $0,5 \%(\mathrm{~m} / \mathrm{v})$ por $12 \mathrm{~h}$ (Vijaya et al., 2008). A cura foi realizada pelo método físicoquímico. As esferas foram secas em estufa a $50{ }^{\circ} \mathrm{C}$ por $12 \mathrm{~h}$ e na sequencia foram imersas em solução de $\mathrm{NaOH} 1,0$ mol.L $\mathrm{L}^{-1}$ por $4 \mathrm{~h}$, a uma temperatura de $25 \pm 2{ }^{\circ} \mathrm{C}$ (Vieira et al., 2014). Ao final, faz-se uma lavagem nas esferas recobertas para a retirada do excesso de reagentes e as mesmas são acondicionadas em potes hermeticamente fechados. A massa de quitosana aderida nas esferas foi medida através da pesagem das esferas antes e após o recobrimento.

\subsection{Ensaios de Equilíbrio}

As soluções foram preparadas em balão volumétrico $(100 \mathrm{~mL})$, no qual foram adicionados $10 \mathrm{~mL}$ de tampão Mcllvaine para correção do $\mathrm{pH}(\mathrm{pH}=3)$ e diferentes volumes da solução de corante $\left(1\right.$ g.L.-1) a fim de variar a concentração inicial de 100 a 700 mg.L $\mathrm{L}^{-1}$ e
Comentar [U]: As temperaturas de estudo foram estipuladas através de informações de trabalhos científicos e trabalhos anteriores.

Comentar [U]: O corante foi considerado puro. Os $10 \%$ restantes são compostos por substâncias inorgânicas que não influenciam na adsorção. 
completou-se o volume com água destilada. Transferiu-se esta solução para os recipientes com as esferas recobertas com quitosana (50 mg quitosana em $22 \mathrm{~g}$ de esferas) e estes foram mantidas em incubadora por $24 \mathrm{~h}$ nas temperaturas (293, 303 e $313 \mathrm{~K})$. Estes ensaios foram realizados em triplicata. A concentração do corante remanescente foi determinada por espectrofotometria (Quimis, modelo Q108 DRM, Brasil) (Dotto et al., 2011). A capacidade de adsorção no equilíbrio $\left(\mathrm{q}_{\mathrm{e}}\right)$ foi determinada pela Equação 1.

$$
q_{e}=\frac{C_{0}-C_{e}}{m} V
$$

na qual, $\mathrm{C}_{0}$ e $\mathrm{C}_{\mathrm{e}}$ são as concentrações inicial e de equilíbrio na fase líquida (mg.L $\left.\mathrm{L}^{-1}\right)$, $\mathrm{m}$ é a massa de adsorvente (g) e V é o volume da solução (L).

Para correlacionar os dados experimentais de equilíbrio utilizou-se o modelo de isotermas de BET. Este modelo considera várias camadas adsorvidas e obedece às seguintes restrições: (a) A adsorção ocorre em várias camadas independentes e imóveis; (b) O equilíbrio é alcançado para cada camada; (c) Além da primeira camada, a adsorção é aproximadamente igual à precipitação. A isoterma de BET é representada pela Equação 2.

$$
q_{e}=\frac{q_{B E T} k_{1} C_{e}}{\left(1-K_{2} C_{e}\right)\left(1-K_{2} C_{e}+K_{1} C_{e}\right)}
$$

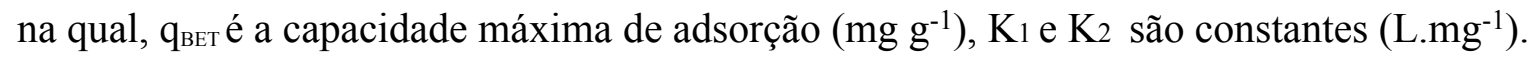

As variações da energia de Gibbs $(\Delta \mathrm{G})$, entalpia $(\Delta \mathrm{H})$ e entropia $(\Delta \mathrm{S})$ foram estimadas a partir das Equações 3 e 4, respectivamente (Milonjic, 2007):

$$
\begin{aligned}
& \Delta G=-R T \ln \left(\rho K_{D}\right) \\
& \ln \left(\rho K_{D}\right)=-\frac{\Delta H}{R T}+\frac{\Delta S}{R}
\end{aligned}
$$

nas quais, $R$ é a constante universal dos gases $\left(8,314 \mathrm{~J} \cdot \mathrm{mol}^{-1} \cdot \mathrm{K}^{-1}\right)$, T é a temperatura $(\mathrm{K}), \rho$ é a densidade da água $\left(\mathrm{g} \mathrm{L}^{-1}\right)$ e $\mathrm{K}_{\mathrm{D}}$ é a constante de equilíbrio termodinâmico (L.g $\left.{ }^{-1}\right)$. $\mathrm{O} \mathrm{k}_{\mathrm{D}}$ foi calculado a partir da declividade da curva de equilíbrio, considerando a primeira parte linear da curva de equilíbrio (Piccin et al.,2012).

\section{RESULTADOS E DISCUSSÕES}

\subsection{Isotermas de equilíbrio e termodinâmica}

A quitosana utilizada nos ensaios de adsorção formou uma camada homogênea sobre a superfície da esfera com $2,633 \mathrm{mg}_{\text {quitosana }} / \mathrm{g}_{\text {esfera }}$. As isotermas de equilíbrio foram construídas na faixa de 293 a $313 \mathrm{~K}$ com o intuito de verificar o efeito da temperatura. A Figura 2 apresenta os dados experimentais de equilíbrio nas temperaturas estudadas para adsorção do corante tartrazina por esferas recobertas com quitosana. 
Figura 2 - Isotermas de equilíbrio de adsorção para o corante tartrazina.

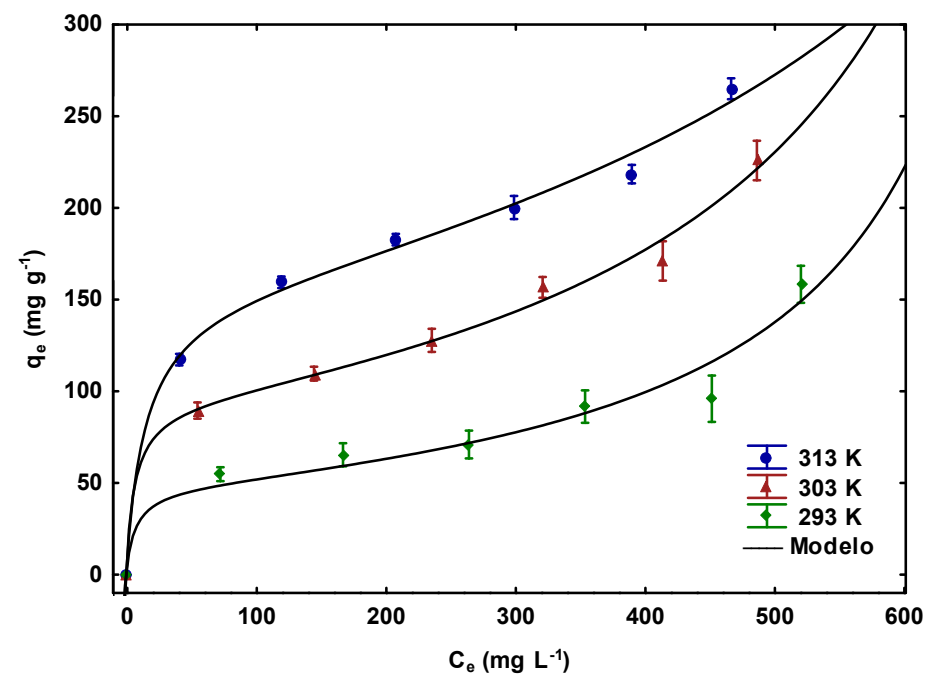

Pode-se observar na Figura 2, que independentemente da condição, as curvas apresentaram um formato sigmoide, em que as isotermas são caracterizadas por um aumento gradual na capacidade de adsorção. Desta forma, distinguem-se bem as etapas de adsorção na monocamada e multicamadas. Segundo Piccin et al. (2012) este comportamento se deve a um efeito sinérgico em que as moléculas adsorvidas facilitam a adsorção de moléculas adicionais devido as interações entre adsorbato-adsorbato. Este processo de adsorção em que ocorre a formação de multicamadas caracteriza as isotermas como do tipo II (Blázquez et al., 2010), as quais, usualmente têm seus dados experimentais de equilíbrio representados pelo modelo de isoterma de BET. A Tabela 1 apresenta os parâmetros das isotermas e a qualidade do ajuste dos dados experimentais ao modelo de BET. A partir dos valores da Tabela $1\left(\mathrm{R}^{2}>0,95\right.$ e EMR $<5 \%$ ) pode-se inferir que o modelo de BET foi adequado para representar os dados experimentais de equilíbrio.

Tabela 1 - Parâmetros de isotermas para a adsorção do corante tartrazina por esferas recobertas com quitosana.

\begin{tabular}{cccc}
\hline Isoterma & $\mathbf{2 9 3}$ & Temperatura (K) & $\mathbf{3 1 3}$ \\
\hline BET & & $\mathbf{3 0 3}$ & \\
$\mathrm{q}_{\mathrm{BET}}\left(\mathrm{mg} \mathrm{g}^{-1}\right)$ & 47,8 & 93,3 & 153,3 \\
$\mathrm{~K}_{1}\left(\mathrm{mg} \mathrm{L}^{-1}\right)$ & 0,173 & 0,156 & 0,070 \\
$\mathrm{~K}_{2}\left(\mathrm{mg} \mathrm{L}^{-1}\right)$ & 0,00131 & 0,00119 & 0,00089 \\
$\mathrm{R}^{2}$ & 0,96 & 0,99 & 0,99 \\
$\mathrm{EMR}^{(\%)}$ & 4,54 & 2,18 & 2,22 \\
\hline
\end{tabular}

Em relação ao efeito da temperatura, pode-se verificar na Figura 2 e na Tabela 1 que na temperatura de $313 \mathrm{~K}$ o corante foi mais fortemente adsorvido pela quitosana aderida nas esferas, desta forma, a elevação da temperatura ocasionou o aumento do valor de $\mathrm{q}_{\mathrm{BET}}$. Além disso, os valores de capacidade máxima de adsorção de BET são condizentes com os valores experimentais de capacidade de adsorção. Estes foram de 54,8 mg.g $\mathrm{g}^{-1}, 89,5 \mathrm{mg} \cdot \mathrm{g}^{-1}$ e 159,5 


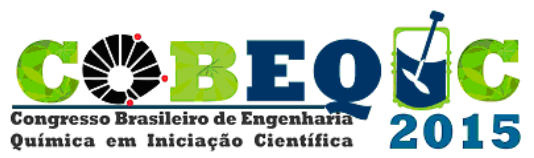

XI Congresso Brasileiro de Engenharia Química em Iniciação Científica Unicamp - Campinas - SP 19 a 22 de julho de 2015

mg. $\mathrm{g}^{-1}$ para as temperaturas de $293 \mathrm{~K}, 303 \mathrm{~K}$ e $313 \mathrm{~K}$, respectivamente. Cheung et al. (2009) obtiveram $113,2 \mathrm{mg} . \mathrm{g}^{-1}$ utilizando nanopartículas de quitosana na remoção de corantes ácidos.

O comportamento termodinâmico $(\Delta \mathrm{G}, \Delta \mathrm{H}$ e $\Delta \mathrm{S})$ da adsorção do corante tartrazina por esferas recobertas com quitosana está apresentado na Tabela 2. O $\Delta \mathrm{G}$ foi obtido através da Equação 3, o $\Delta \mathrm{H}$ e $\Delta \mathrm{S}$ foram determinados através do gráfico de Van't Hoff's , ajustando os dados a Equação 4.

Analisando a Tabela 2, pode-se verificar a natureza endotérmica da adsorção pelo valor positivo de $\Delta H$. De acordo com Cardoso et al. (2011), valores de $\Delta H$ na faixa de 40 a $800 \mathrm{~kJ} \mathrm{~mol}^{-1}$ são característicos de processos que envolvem quimiossorção. Os valores negativos de $\Delta \mathrm{G}$ mostram que a adsorção do corante tartrazina por esferas recobertas com quitosana foi um processo espontâneo e favorável. Além disso, pode-se verificar que a desordem do sistema aumentou durante a adsorção.

Tabela 2 - Parâmetros termodinâmicos para a adsorção.

\begin{tabular}{cccc|}
\hline $\mathrm{T}(\mathrm{K})$ & $\Delta \mathrm{G}\left(\mathrm{kJ} \mathrm{mol}^{-1}\right)$ & $\Delta \mathrm{H}\left(\mathrm{kJ} \mathrm{mol}^{-1}\right)$ & \multicolumn{2}{c}{$\Delta \mathrm{S}\left(\mathrm{kJ} \mathrm{mol}^{-1} \mathrm{~K}^{-1}\right)$} \\
\hline 293 & $-11,4$ & & \\
303 & $-13,6$ & 40,4 & $0,177^{----7^{2}}$ \\
313 & $-14,9$ & & \\
\hline
\end{tabular}

\section{4. (CONCLUSÃO)}

Neste trabalho foram estudadas as isotermas e a termodinâmica da adsorção do corante tartrazina por esferas recobertas com quitosana em diferentes temperaturas. A capacidade de adsorção foi aumentada com o aumento da temperatura. $\mathrm{O}$ modelo de BET foi apropriado para representar os dados experimentais de equilíbrio $\left(\mathrm{R}^{2}>0,95\right.$ e $\left.\mathrm{EMR}<5 \%\right)$. A máxima capacidade de adsorção na monocamada foi de $153,3 \mathrm{mg} \cdot \mathrm{g}^{-1}$ à $313 \mathrm{~K}$, mostrando que as esferas recobertas com quitosana podem viabilizar o uso da quitosana no processo de adsorção em leito fixo pois permitem a flexibilidade de design e engenharia necessárias. Isto se deve ao fato da alta capacidade de adsorção das esferas recobertas por quitosana e da eliminação das limitações hidrodinâmicas do sistema. O estudo termodinâmico mostrou que a adsorção do corante tartrazina por esferas recobertas com quitosana foi um processo espontâneo, favorável, endotérmico, e que a desordem do sistema aumentou durante a adsorção. A magnitude de $\Delta \mathrm{H}$ mostrou que a quimiossorção teve um papel importante na adsorção do corante.

\section{REFERÊNCIAS}

BLÁZQUEZ, G.; CALERO, M.; HERNÁINZ, F.; TENORIO, G.; MARTÍN-LARA, M. A. Equilibrium biosorption of lead(II) from aqueous solutions by solid waste from oliveoil production, Chem. Eng. J., v.160, p. 615-622, 2010.

CARDOSO, N. F.; PINTO, R. B.; LIMA, E. C.; CALVETE, T.; AMAVISCA, C. V.; ROYER, B.; CUNHA, M. L.; FERNANDES, T. H. M.; PINTO, I. S. Removal of remazol black B textile dye from aqueous solution by adsorption. Desalination., v. 269, p. $92-103,2011$.
Comentar [U]: Para o cálculo da entalpia e entropia o método de Van't Hoff's não considera a variação de temperatura dentro da faixa estabelecida. Através da Eq. 4 são obtidos o coeficiente angular e linear da reta.

Comentar [U]: Para ter conclusões mais expressivas sobre o tratamento em escala industrial devem ser realizados estudos aplicando as esferas recobertas em coluna de leito fixo. Este é o próximo passo no desenvolvimento do trabalho. 
CHEUNG, W. H.; SZETO, Y. S.; McKAY, G. Enhancing the adsorption capacities of acid dyes by chitosan nano particles., Bioresour. Technol., v. 100, p. $1143-1148,2009$.

CHIOU, M. ; HO, P.; LI, H. Adsorption of anionic dyes in acid solutions using chemically cross-linked chitosan beads, Dyes and Pigm., v. 60, p. 69 - 84, 2004.

CRINI, G.; BADOT, P. Application of chitosan, a natural aminopolysaccharide, for dye removal from aqueous solutions by adsorption processes using batch studies: A review of recent literature. Prog. Polym. Sci., v. 33, p. 399 - 447, 2008.

DOTTO, G. L.; VIEIRA, M. L. G.; GONÇALVES, J. O.; PINTO, L. A. A. Remoção dos corantes azul brilhante, amarelo crepúsculo e amarelo tartrazina de soluções aquosas utilizando carvão ativado, terra ativada, terra diatomácea, quitina e quitosana: Estudos de equilíbrio e termodinâmica. Química Nova., v. 34, p. 1193 - 1199, 2011.

MILONJIC, S. K. A consideration of the correct calculation of thermodynamic parameters of adsorption, J. Serb. Chem. Soc., v. 72, p. 1363 - 1367, 2007.

MOUSSAVI, G.; MAHMOUDI, M. Removal of azo and anthraquinone reactive dyes from industrial wastewaters using $\mathrm{MgO}$ nanoparticles, J. Hazard. Mater,. v. 168, p. $806-$ $812,2009$.

PICCIN, J. S.; VIEIRA, M. L. G.; DOTTO, G. L.; PINTO, L. A. A. Adsorption of FD\&C Red No, 40 by chitosan: Isotherms analysis, J. Food Eng., v. 95, p. 16 - 20, 2009.

PICCIN, J.S.; GOMES, C.S.; FERIS, L.A.; GUTTERRES, M. Kinetics and isotherms of leather dye adsorption by tannery solid waste. Chem. Eng. J., v. 183, p. 30 - 38, 2012.

SHIRZAD-SIBONI, M.; JAFARI, S. J.; GIAHI, O.; KIM, I.; LEE, S.; YANG, J. Removal of acid blue 113 and reactive black 5 dye from aqueous solutions by activated red mud, $J$. Ind. Eng. Chem., v. 20, p. 1432 - 1437, 2014.

SRINIVASAN, A.; VIRARAGHAVAN, T. Decolorization of dye wastewaters by biosorbents: A review, J. Environ. Manage., v. 91, p. 1915 - 1929, 2010.

VIEIRA, M. L. G.; ESQUERDO, V. M.; NOBRE, L. R.; DOTTO, G. L.; PINTO, L. A. A. Glass beads coated with chitosan for the food azo dyes adsorption in a fixed bed column, J. Ind. Eng. Chem., v. 20, p. 3387 - 3393, 2014.

VIJAYA, Y.; POPURI, S. R.; BODDU, V. M., KRISHNAIAH, A. Modified chitosan and calcium alginate biopolymer sorbents for removal of nickel (II) through adsorption, Carbohydr. Polym., v. 72, p. $261-271,2008$. 